\title{
Packaging Contains Unintended Material
}

National Cancer Institute

\section{Source}

National Cancer Institute. Packaging Contains Unintended Material. NCI Thesaurus. Code C139504.

Problems that occurred because unintended material was packaged with the device. 